\title{
Comparison of T1rho imaging between spoiled gradient echo (SPGR) and balanced steady-state free precession (b-FFE) sequence of knee cartilage at 3 tesla MRI
}

\author{
Taiki Nozaki, M.D1, Yasuhito Kaneko, M.D, PhD' ${ }^{1}$, Hon J.Yu, PhD' ${ }^{1}$, Kayleigh Kaneshiro², \\ Ran Schwarzkopf, M.D, M.Sc. ${ }^{3}$, and Hiroshi Yoshioka, M.D, PhD' \\ ${ }^{1}$ Department of Radiological Sciences, University of California, Irvine, CA, USA \\ ${ }^{2}$ Medical School, University of California, Irvine, CA, USA \\ ${ }^{3}$ Department of Orthopaedic Surgery, University of California, Irvine, CA, USA
}

\section{Abstract}

Purpose-To investigate the difference in T1rho profiles of the entire femoral cartilage between SPGR and b-FFE sequences at 3.0T.

\begin{abstract}
Materials and methods-20 healthy volunteers were enrolled in this study. T1rho images of each subject were acquired with two types of pulse sequences: SPGR and b-FFE. Femoral cartilage segmentation was performed by two independent raters slice-by-slice using Matlab. Inter- and intra- observer reproducibility between the two imaging protocols was calculated. The relative signal intensity (SI) of cartilage, subchondral bone marrow, joint effusion, and the relative signal contrast between structures of the knee were quantitatively measured. The difference in T1rho values between SPGR and b-FFE sequences was statistically analyzed using the Wilcoxon signed-rank test.
\end{abstract}

Results-The average T1rho value of the entire femoral cartilage with b-FFE was significantly higher compared to SPGR $(\mathrm{p}<0.05)$. The reproducibility of the segmented area and T1rho values was superior with SPGR compared to b-FFE. The inter-class correlation coefficient was 0.846 on SPGR and 0.824 on b-FFE. The intra-class correlation coefficient of T1rho values was 0.878 on SPGR and 0.836 on b-FFE. The two imaging techniques demonstrated different signal and contrast characteristics. The relative SI of fluid was significantly higher on SPGR, while the relative SI of subchondral bone was significantly higher on b-FFE $(p<0.001)$. There were also significant differences in the relative contrast between fluid-cartilage, fluid-subchondral bone, and cartilage-subchondral bone between the two sequences (all $\mathrm{p}<0.001$ ).

Conclusion-We need to pay attention to differences in T1rho values between SPGR and b-FFE in clinical applications.

\section{Keywords}

T1rho; femoral cartilage; SPGR; b-FFE; 3.0T-MRI

Address correspondence to: Hiroshi Yoshioka M.D. Ph.D., Professor in Residence, Radiological Sciences, University of California, Irvine, 101 The City Drive South, Route 140, Orange, CA 92868, Phone; 714-456-8849, Fax; 714-456-7864, hiroshi@uci.edu. 


\section{Introduction}

Osteoarthritis (OA) is one of the most prevalent disorders in today's society, resulting in significant socio-economic costs and morbidity [1]. A host of new and exciting therapeutic modalities are being developed for the treatment of OA, including new chondroprotective and chondroregenerative drugs, osteochondral autografting, and autologous chondrocyte implantation. Therefore, it is important to detect early cartilage degeneration and understand its natural progression in order to treat OA.

Novel MRI pulse sequences for cartilage assessment have recently received increased attention due to newly developed protocols for physiologic MRI which include T2 mapping and T1rho mapping [2,3]. By providing information about the interactions between motionrestricted water molecules and their local macromolecular environment within cartilage. With these techniques, we are able to assess the degree of degeneration in cartilage. Increases in the $\mathrm{T} 2$ relaxation time of cartilage have been associated with matrix damage, particularly with the loss of collagen integrity. In contrast, the T1rho relaxation rate in cartilage decreases linearly with the percentage of proteoglycan loss [4,5]. T1rho mapping has been shown to be more sensitive to biochemical change in cartilage than T2 mapping, and this has enabled early detection of cartilage degeneration in early OA patients before gross morphological change [6,7].

However, it is not well understood what can be considered as a normal range of T1rho values. Furthermore, multiple factors affect T1rho mapping such as the specific MR protocol used or the post-imaging processing, including the methodology of operatordependent manual cartilage segmentation. Many reports have recently been published regarding the T1rho values of both healthy and damaged knee cartilage, although the MRI protocols and methodology of segmentation vary among these reports (Table 1) [8-21]. Regarding MRI sequences, there are two basic types of fast gradient echo (GRE) sequences used in T1rho mapping. One is a spoiled GRE sequence; residual transverse magnetization is spoiled. The other is a steady-state GRE sequence; transverse magnetization is not spoiled but is refocused to contribute to steady-state formation [22]. There is no prior study that compares these two sequences. For clinical applications and comparison of these results with prior studies, it is important to understand the characteristics and differences between the two sequences.

Therefore, the purpose of this study is to investigate the reproducibility of cartilage segmentation and evaluate the difference in T1rho profiles between the spoiled gradient echo (SPGR) and balanced steady state free precession (b-FFE) sequences.

\section{Materials and Methods}

\section{Subjects}

23 healthy volunteers (mean: 28.9 years, range: 19-38) were recruited in this study. Inclusion criteria for all subjects included: patients 18-40 years of age with no knee symptoms and no history of prior knee surgery. We excluded 3 subjects from the study, including one who had a large knee that couldn't fit within the knee coil, and two patients 
who were claustrophobic. In total, 20 healthy volunteers were studied. The study was approved by the institutional review board (IRB) and conformed to the Declaration of Helsinki. Written informed consent was obtained from each subject.

\section{MR imaging protocol}

All MR studies were performed on a 3.0-T unit (Achieva, Philips Healthcare, Netherland) utilizing an 8-channel knee receive-only RF-coil. Two sagittal T1rho-weighted images of each subject were acquired on the pulse sequence of b-FFE and SPGR. All sagittal images were obtained without oblique angulation, parallel to the magnetic static field (B0). The acquisition parameters were as follows. SPGR: mode $=3 \mathrm{D}$, fat-saturation method $=$ PROSET, TR $/ \mathrm{TE}=6.4 / 3.4 \mathrm{msec}$, Band width $=475 \mathrm{~Hz}, \mathrm{ETL}=64, \mathrm{NEX}=1, \mathrm{FOV}=$ $140 * 140 \mathrm{~mm}$, Slice thickness $/$ gap $=3 / 0 \mathrm{~mm}$, Flip angle $=10$ degree, Image-matrix $=$ $512 * 512 \mathrm{~mm}$, number of slices $=31$, effective in-plane spatial resolution $=0.27 * 0.27 \mathrm{~mm}$, acceleration factor $=2$, spin-lock frequency $=575 \mathrm{~Hz}$, Time of spin-lock $(\mathrm{TSL})=$ 20/40/60/80msec, acquisition time $=4 \min 09 \mathrm{sec} * 4$, and b-FFE: mode $=3 \mathrm{D}$, fat-saturation method $=$ SPIR, TR $/ \mathrm{TE}=4.8 / 2.4 \mathrm{msec}$, Band width $=606 \mathrm{~Hz}, \mathrm{ETL}=154, \mathrm{NEX}=1, \mathrm{FOV}=$ $140 * 140 \mathrm{~mm}$, Slice thickness $/$ gap $=3 / 0 \mathrm{~mm}$, Flip angle $=25$ degree, Image-matrix $=$ $512 * 512 \mathrm{~mm}$, number of slices $=31$, effective in-plane spatial resolution $=0.27 * 0.27 \mathrm{~mm}$, spin-lock frequency $=575 \mathrm{~Hz}$, Time of spin-lock $(T S L)=20 / 40 / 60 / 80 \mathrm{msec}$, acquisition time $=3 \mathrm{~min} 57 \mathrm{sec} * 4$. Parallel imaging was used on all imaging sequences utilizing Sensitivity Encoding (SENSE) with an acceleration factor of 2.

\section{Image processing and cartilage segmentation of entire knee}

Images were transferred in DICOM (Digital Imaging and Communications in Medicine) format to a personal computer (PC; Windows 7), which was used to perform all postprocessing and analyses. For possible motion between the scans, the T1rho series were first realigned with respect to the first TSL images using rigid-body transformation before being fitted to a mono-exponential function on a pixel-by-pixel basis for generation of T1rho maps: $\mathrm{S}(\mathrm{TSL})=\mathrm{S} 0 * \exp (-\mathrm{TSL} / \mathrm{T} 1 \mathrm{rho})$, where $\mathrm{S} 0$ is the signal intensity when TSL $=0$. Manual cartilage segmentation of the entire knee was then independently performed sliceby-slice for each T1rho image by a board-certified orthopaedic surgeon (R1) and a boardcertified radiologist sub-specialized in musculoskeletal radiology (R2). Images of $\mathrm{TSL}=20 \mathrm{msec}$ were chosen for cartilage segmentation as they had the highest signal to noise ratio (SNR). Once the cartilage was segmented by manually placing vertices along their boundary, their $\mathrm{x}$ and $\mathrm{y}$ coordinates were used in a circle fitting algorithm by assuming a circular cartilage shape about an imaginary center position in the subchondral bone. The coordinates of this center point were estimated in a least squares manner. The user could also manually choose the center point if necessary. Additional boundary vertices with finer spacing were then interpolated and used for computation of slope angles for the radial vectors from the center position to each boundary vertex. For each slope angle (in step of 1degree) the farthest (closest to the articular surface) and nearest (closest to the bone) boundary vertices were recorded while the radial points between the boundary vertices were approximated by a linear interpolation and recorded for subsequent depth/angle-based segmentation of cartilage. The T1rho depth/angle-dependent profile was investigated in this study by partitioning cartilage into two layers (deep; 51-100\% and superficial; 0-50\%) and 
angular segmentations in step of 4-degree over the length of segmented cartilage (with the angle 0 defined along B0) with positive/negative angles by a counter-clockwise/clockwise rotation (Fig.1). All image processing described above was performed using an in-house developed and implemented software run in Matlab (Mathworks, Natick, MA, USA).

\section{Analysis of T1 rho value differences between SPGR and b-FFE sequences}

We calculated the average T1rho values of the entire femoral cartilage in both the superficial and deep layer with a 4-degree stepwise analysis on both the SPGR and b-FFE T1rho sequences, and compared the difference in T1rho values between these two sequences.

Regarding T1rho values and segmented areas, the inter- and intra-observer reproducibility of cartilage segmentation of the two T1rho sequences was measured.

\section{Quantitative analysis with relative SI and relative contrast between two $\mathrm{T} 1$ rho sequences}

A board-certified radiologist (R2) measured the mean and standard deviation (SD) of signal intensity (SI) of the cartilage, joint fluid, and subchondral bone of the knee, placing regions of interest (ROI) in each of the 20 knees, utilizing the same method as previously reported (23). The ROIs were identical in size and placed in identical positions on matching sections (Fig.2). We used relative SI and relative contrast for direct comparison of image quality between the SPGR and b-FFE images because all of the sequences in our study were obtained with a parallel imaging technique. The relative SI of each structure was calculated as SI/SD. Relative contrast of "structure A" to "structure B" was defined as $\left(\mathrm{SI}_{\mathrm{A}}-\mathrm{SI}_{\mathrm{B}}\right) /\left(\mathrm{SD}_{\mathrm{A}}{ }^{2}\right.$ $\left.+\mathrm{SD}_{\mathrm{B}}^{2}\right)^{1 / 2}$.

\section{Statistical Analysis}

The difference in T1rho values between SPGR and b-FFE sequences was statistically analyzed using the Wilcoxon signed-rank test. Comparison of relative SI and the relative contrast between the two T1rho sequences was also analyzed using the Mann-Whitney test.

Inter- and intra-observer reliability and the variability of T1rho values between the two imaging protocols were calculated as inter- and intra-class correlation coefficients. For the evaluation of the reproducibility of the segmented area (pixels) between two operators and intra-operators, we performed Bland-Altman plot analysis.

Statistical analyses were performed using the $\mathrm{R}$ version 3.0.2 for Windows software ( $\mathrm{R}$ Development Core Team, Vienna, Austria), except for the BA-plots analyses of segmented areas for inter-rater and intra-rater reproducibility. For these, we used Matlab for analysis.

\section{Results}

Inter- and intra-observer reproducibility of manual segmentation between SPGR and b-FFE sequences

Table 2 summarizes the inter- and intra-observer reproducibility regarding the T1rho values and segmented area in each layer. For measurements of average T1rho values of the superficial layer of the entire femoral cartilage, inter-class and intra-class correlation 
coefficients were lower on SPGR than b-FFE, while in the deep layer, they were higher on SPGR than b-FFE. In total, the inter-class correlation coefficient in the whole layer was higher on SPGR (0.846) than b-FFE (0.824). The intra-class correlation coefficient in the whole layer was also higher on SPGR (0.878) than b-FFE (0.836).

In Figures 3 and 4, Bland-Altman plots of both readers' measurements are shown for the segmented pixel numbers of the whole layer on SPGR and b-FFE sequences. The correlation coefficient of the segmented area in the whole layer between the two operators was 0.641 on SPGR and 0.588 on b-FFE, and the correlation coefficient between intra-operators was 0.858 on SPGR and 0.796 on b-FFE. The inter- and intra-observer reproducibility of the segmented area was higher on SPGR than b-FFE in both of the superficial and deep layer.

\section{Quantitative analysis with relative signal intensity and relative contrast}

Figure 5 demonstrates the mean values of relative SI and relative contrast for each sequence. The relative SI of cartilage was $11.9 \pm 4.2($ mean \pm SD) on SPGR and $13.0 \pm 6.8$ on b-FFE, without a statistically significant difference. The relative SI of joint fluid was higher on SPGR $(22.0 \pm 7.0)$ than b-FFE $(10.5 \pm 6.1)(\mathrm{p}<0.001)$, while that of subchondral bone was higher on b-FFE $(6.3 \pm 2.0)$ than SPGR $(2.3 \pm 0.6)(\mathrm{p}<0.001)$. The relative contrast of fluidcartilage was a positive value of $4.5 \pm 1.6$ on SPGR and a negative value of $-3.5 \pm 3.3$ on $b-$ FFE. The relative contrast of cartilage-subchondral bone on SPGR $(8.8 \pm 2.6)$ was higher than b-FFE (6.3 \pm 2.9$)$. There were significant differences in fluid-cartilage, fluid-subchondral bone, and cartilage-subchondral bone relative contrast between the two sequences (all $\mathrm{p}<0.001)$.

\section{Analysis of $\mathrm{T} 1$ rho profiles}

Figure 6 compares the T1rho profiles of each layer on both sequences. T1rho values tended to be higher on the b-FFE sequence compared to the SPGR sequence in all layers, more predominantly in the deep layer between approximately -70 and +70 degrees. There was angular variation of T1rho profiles in each layer on both sequences. For the b-FFE sequence, there was a peak of T1rho values at approximately $+20-30$ degrees. The average T1rho value of the femoral cartilage in the whole layer was $57.0 \pm 2.8$ on SPGR and $59.5 \pm 2.8$ on b-FFE, a statistically significant difference $(\mathrm{p}<0.05)$. In the superficial layer, it was $58.9 \pm 2.6$ on SPGR and $60.5 \pm 3.5$ on b-FFE. In the deep layer, it was 55.7 \pm 3.6 on SPGR and 59.1 \pm 2.8 on b-FFE. Average T1rho values in the superficial layer of the entire femoral articular cartilage were higher than in the deep layer $(\mathrm{p}<0.05)$ on both sequences.

\section{Discussion}

In this study, we demonstrated the difference in T1rho values between SPGR and b-FFE sequences and the reproducibility of segmentation in these sequences for the entire femoral articular cartilage. Regarding the T1rho values, inter- and intra-observer reproducibility was excellent on both sequences. However, the reproducibility of segmented area by pixel counts was superior on SPGR compared to b-FFE for all layers. This suggests that the outline between subchondral bone and cartilage, and between fluid and cartilage is more distinct on SPGR, because relative contrasts between each structure are superior on the SPGR 
sequence. However, in the superficial layer, the inter- and intra-class correlation coefficients of the T1rho values were higher on b-FFE than on SPGR. We suppose that one of the reasons for the lower inter- and intra-class correlation coefficients for SPGR in the superficial layer is due to the large differences in T1rho values between fluid and cartilage in this sequence. Small areas of contamination from high signal fluid can more easily influence the mean cartilage T1rho value for SPGR. In this study, the SPGR sequence demonstrated positive fluid-cartilage contrast (fluid signal was higher than cartilage signal), while b-FFE demonstrated negative fluid-cartilage contrast (fluid signal was lower than cartilage signal). Negative contrast between fluid and cartilage on b-FFE may better delineate the cartilage surface. In contrast, in the deep layer, the inter- and intra-class correlation coefficients of the T1rho values were higher on SPGR compared to b-FFE. We suppose that this is due to the relatively superior cartilage-to-subchondral bone contrast of SPGR compared to b-FFE, with significant difference by combination of high relative SI of cartilage and low relative SI of subchondral bone. All in all, in the whole layer analysis, SPGR is superior to b-FFE in both inter- and intra-reproducibility.

In general, the advantages of steady-state sequences are better SNR and contrast-to-noise ratio (CNR) compared to spoiled GRE sequences [22]. Steady-state sequences can be classified as three types [24]. That is, post-excitation refocused steady-state sequences, preexcitation refocused steady-state sequences, and fully refocused steady-state sequences. Balanced FFE is one of the fully refocused steady-state sequences, and the main difference from other GRE sequences is the usage of balanced gradients in all three axes (sliceselection, phase-encoding, and readout). This makes refocused steady-state sequences relatively insensitive to motion [25]. Contrast in this sequence is dependent on the T2/T1 ratio, and a high SNR is another advantage of this sequence. For these reasons, this sequence is mainly used in cardiac imaging and fetal imaging $[22,26]$. However, a fully refocused steady-state sequence has the disadvantage of banding artifacts, especially at air-tissue interfaces caused by field inhomogeneity [26]. Although a spoiled GRE sequence is sensitive to motion, fast T1-weighted images can be acquired on a spoiled GRE sequence. Fat-suppressed three dimensional SPGR is not only suitable for detection of articular cartilage lesions, but also for measurement of cartilage volume and surface area measurement, with excellent longitudinal reproducibility due to a high CNR between cartilage and surrounding tissues [27-29].

In the analysis of depth dependence, it is important to note that cartilage has an organized layered structure divided into four zones; the superficial zone, the middle zone, the deep zone, and the zone of calcified cartilage. The chondrocytes in the superficial layer secrete relatively little proteoglycan. In contrast, the deep layer contains the highest proteoglycan content. Proteoglycans resist compression and generate swelling pressure due to their affinity for water [30]. Our results are concordant with these facts, as the T1rho values of the superficial layers were higher than those of the deep layers over the entire femoral condyle.

In a previously published study, mean T1rho values over the medial femoral condyle of normal subjects were 41.9 (msec) in the whole layer, 47.2 (msec) in the surface layer, and $36.4(\mathrm{msec})$ in the deep layer on the SPGR sequence [9]. Another study has reported that the mean T1rho values on a balanced turbo field echo sequence over the medial and lateral 
femoral condyles of normal subjects was $53.0(\mathrm{msec})$ and $53.3(\mathrm{msec})$ in the weight-bearing portion and 49.9 (msec) and $51.1(\mathrm{msec})$ in the less-weight-bearing portion [10]. These T1rho values from previous studies are lower than our results, although we cannot compare these results directly due to differences in segmentation method, ROI setting, inclusion criteria of control subjects, imaging sequences, and vendors. Clinically, it is important to understand the difference in T1rho values between different MR sequences and between different vendors' MR machines. When comparing with previous reports, attention must be paid to the type of T1rho sequence used.

In the orientation analysis, angular variation of T1rho profiles was seen in each layer on both sequences. T1rho values in the weight-bearing portion (approximately $-30-/+30$ degrees) vary by angular location on both sequences, especially in the deep layer. In general, T1rho values decrease as proteoglycan content increases. Therefore, our results suggest that the proteoglycan content is not constant, even within the weight-bearing portion, with a higher proteoglycan content in the anterior weight-bearing portion. When the angle from B0 exceeded \pm 100 degrees, the difference in T1rho values between the two sequences was negligible. Cartilage T1rho values between the two sequences converge near the anterior and posterior portion of the femoral condyle. The reason for this is unclear, however it may be due to differences in sensitivity to proteoglycan content between the two sequences. If the sensitivity to proteoglycan is higher on SPGR than b-FFE, the difference in T1rho values between the two sequences will be larger in the weight-bearing portion, and it will be smaller in the less-weight-bearing portion. Alternatively, since bone marrow signal on bFFE is bright, chemical shift artifact may influence T1rho values more in the deep layer on this sequence, with a greater effect on the central portion of the femoral condyle, and a lesser effect on the anterior or posterior portion of the femoral condyle.

Our study has several limitations. First, there is no gold or reference standard for T1rho values of the entire femoral cartilage. Therefore, it is difficult to assess the accuracy of our segmentation. Because partial volume effect is inevitable for a curved cartilage structure in the segmentation of the entire femoral cartilage, it is difficult to extract cartilage precisely, especially at the anterior and posterior cartilage borders. Contamination of subchondral bone and fluid may also be considerable in these areas. Second, we did not investigate the difference in T1rho values between vendors or machines for the same type of imaging sequence. For example, FIESTA, True FISP and b-FFE are all acronyms of the balanced steady-state free precession sequence. In order to assess for the differences between vendors, it would be necessary to perform MRIs for each subject on each vendor's machine. Third, the superficial and deep layers we analyzed do not correspond to four physiologic layers [30]. Insufficient spatial resolution is a technical limitation that prevents us from distinguishing four distinct physiologic layers. Fourth, there was no comparison available with histological specimens, arthroscopic and biological examination such as the content of proteoglycan. Finally, we utilized two T1rho sequences with long acquisition times. This might increase susceptibility to motion artifact or subtle shifts in knee position between sequences, although there were no subjects with obvious motion artifact or knee position changes in our study. 
In conclusion, inter- and intra-reader reproducibility for femoral cartilage T1rho mapping is excellent on both SPGR and b-FFE sequences, and higher on SPGR than b-FFE. T1rho values tend to be higher on b-FFE compared to SPGR. The practical applications of this study suggest that close attention needs to be paid to the effect that different sequences can have on T1rho values, especially in clinical applications.

\section{References}

1. March LM, Bachmeier CJ. Economics of osteoarthritis: a global perspective. Baillieres Clin Rheumatol. 1997; 11(4):817-34. [PubMed: 9429738]

2. Gold GE, Burstein D, Dardzinski B, Lang P, Boada F, Mosher T. MRI of articular cartilage in OA: novel pulse sequences and compositional/functional markers. Osteoarthritis Cartilage. 2006; 14(Suppl A):A76-86. [PubMed: 16716605]

3. Crema MD, Roemer FW, Marra MD, et al. Articular cartilage in the knee: current MR imaging techniques and applications in clinical practice and research. Radiographics. 2011; 31(1):37-61. [PubMed: 21257932]

4. Goodwin DW, Wadghiri YZ, Dunn JF. Micro-imaging of articular cartilage: T2, proton density, and the magic angle effect. Acad Radiol. 1998; 5(11):790-8. [PubMed: 9809078]

5. Akella SV, Regatte RR, Gougoutas AJ, et al. Proteoglycan-induced changes in T1rho-relaxation of articular cartilage at 4T. Magn Reson Med. 2001; 46(3):419-23. [PubMed: 11550230]

6. Choi JA, Gold GE. MR imaging of articular cartilage physiology. Magn Reson Imaging Clin N Am. 2011; 19(2):249-82. [PubMed: 21665090]

7. Borthakur A, Mellon E, Niyogi S, Witschey W, Kneeland JB, Reddy R. Sodium and T1rho MRI for molecular and diagnostic imaging of articular cartilage. NMR Biomed. 2006; 19(7):781-821. [PubMed: 17075961]

8. Wang L, Vieira RL, Rybak LD, et al. Relationship between knee alignment and T1rho values of articular cartilage and menisci in patients with knee osteoarthritis. Eur J Radiol. 2013; 82(11):194652. [PubMed: 23769189]

9. Schooler J, Kumar D, Nardo L, et al. Longitudinal evaluation of T1rho and T2 spatial distribution in osteoarthritic and healthy medial knee cartilage. Osteoarthritis Cartilage. 2014; 22(1):51-62. [PubMed: 24188868]

10. Goto H, Iwama Y, Fujii M, et al. A preliminary study of the T1rho values of normal knee cartilage using 3T-MRI. Eur J Radiol. 2012; 81(7):e796-803. [PubMed: 22525597]

11. Hirose J, Nishioka H, Okamoto N, et al. Articular cartilage lesions increase early cartilage degeneration in knees treated by anterior cruciate ligament reconstruction: T1rho mapping evaluation and 1-year follow-up. Am J Sports Med. 2013; 41(10):2353-61. [PubMed: 23925576]

12. Takayama Y, Hatakenaka M, Tsushima H, et al. T1rho is superior to T2 mapping for the evaluation of articular cartilage denaturalization with osteoarthritis: radiological-pathological correlation after total knee arthroplasty. Eur J Radiol. 2013; 82(4):e192-8. [PubMed: 23265927]

13. Souza RB, Feeley BT, Zarins ZA, Link TM, Li X, Majumdar S. T1rho MRI relaxation in knee OA subjects with varying sizes of cartilage lesions. Knee. 2013; 20(2):113-9. [PubMed: 23159719]

14. Tsushima H, Okazaki K, Takayama Y, et al. Evaluation of cartilage degradation in arthritis using T1rho magnetic resonance imaging mapping. Rheumatol Int. 2012; 32(9):2867-75. [PubMed: 21881979]

15. Peers SC, Maerz T, Baker EA, et al. T1rho magnetic resonance imaging for detection of early cartilage changes in knees of asymptomatic collegiate female impact and nonimpact athletes. Clin J Sport Med. 2014; 24(3):218-25. [PubMed: 24172654]

16. Bolbos RI, Ma CB, Link TM, Majumdar S, Li X. In vivo T1rho quantitative assessment of knee cartilage after anterior cruciate ligament injury using 3 Tesla magnetic resonance imaging. Invest Radiol. 2008; 43(11):782-8. [PubMed: 18923257]

17. Li X, Kuo D, Theologis A, et al. Cartilage in anterior cruciate ligament-reconstructed knees: MR imaging T1\{rho\} and T2--initial experience with 1-year follow-up. Radiology. 2011; 258(2):50514. [PubMed: 21177392] 
18. Su F, Hilton JF, Nardo L, et al. Cartilage morphology and T1rho and T2 quantification in ACLreconstructed knees: a 2-year follow-up. Osteoarthritis Cartilage. 2013; 21(8):1058-67. [PubMed: 23707754]

19. Theologis AA, Haughom B, Liang F, et al. Comparison of T1rho relaxation times between ACLreconstructed knees and contralateral uninjured knees. Knee Surg Sports Traumatol Arthrosc. 2014; 22(2):298-307. [PubMed: 23370983]

20. Gupta R, Virayavanich W, Kuo D, et al. MR Trho quantification of cartilage focal lesions in acutely injured knees: correlation with arthroscopic evaluation. Magn Reson Imaging. 2014

21. Zarins ZA, Bolbos RI, Pialat JB, et al. Cartilage and meniscus assessment using T1rho and T2 measurements in healthy subjects and patients with osteoarthritis. Osteoarthritis Cartilage. 2010; 18(11):1408-16. [PubMed: 20696262]

22. Chavhan GB, Babyn PS, Jankharia BG, Cheng HL, Shroff MM. Steady-state MR imaging sequences: physics, classification, and clinical applications. Radiographics. 2008; 28(4):1147-60. [PubMed: 18635634]

23. Yamabe E, Anavim A, Sakai T, et al. Comparison between high-resolution isotropic threedimensional and high-resolution conventional two-dimensional FSE MR images of the wrist at 3 tesla: a pilot study. J Magn Reson Imaging. 2014; 40(3):603-8. [PubMed: 24227687]

24. Brown MA, Semelka RC. MR imaging abbreviations, definitions, and descriptions: a review. Radiology. 1999; 213(3):647-62. [PubMed: 10580935]

25. Absil J, Denolin V, Metens T. Fat attenuation using a dual steady-state balanced-SSFP sequence with periodically variable flip angles. Magn Reson Med. 2006; 55(2):343-51. [PubMed: 16402382]

26. Finn JP, Nael K, Deshpande V, Ratib O, Laub G. Cardiac MR imaging: state of the technology. Radiology. 2006; 241(2):338-54. [PubMed: 17057063]

27. Yoshioka H, Stevens K, Hargreaves BA, et al. Magnetic resonance imaging of articular cartilage of the knee: comparison between fat-suppressed three-dimensional SPGR imaging, fat-suppressed FSE imaging, and fat-suppressed three-dimensional DEFT imaging, and correlation with arthroscopy. J Magn Reson Imaging. 2004; 20(5):857-64. [PubMed: 15503323]

28. Disler DG, McCauley TR, Kelman CG, et al. Fat-suppressed three-dimensional spoiled gradientecho MR imaging of hyaline cartilage defects in the knee: comparison with standard MR imaging and arthroscopy. AJR Am J Roentgenol. 1996; 167(1):127-32. [PubMed: 8659356]

29. Brem MH, Pauser J, Yoshioka H, et al. Longitudinal in vivo reproducibility of cartilage volume and surface in osteoarthritis of the knee. Skeletal Radiol. 2007; 36(4):315-20. [PubMed: 17219231]

30. Pearle AD, Warren RF, Rodeo SA. Basic science of articular cartilage and osteoarthritis. Clin Sports Med. 2005; 24(1):1-12. [PubMed: 15636773] 


\section{$A, B$}

SPGR

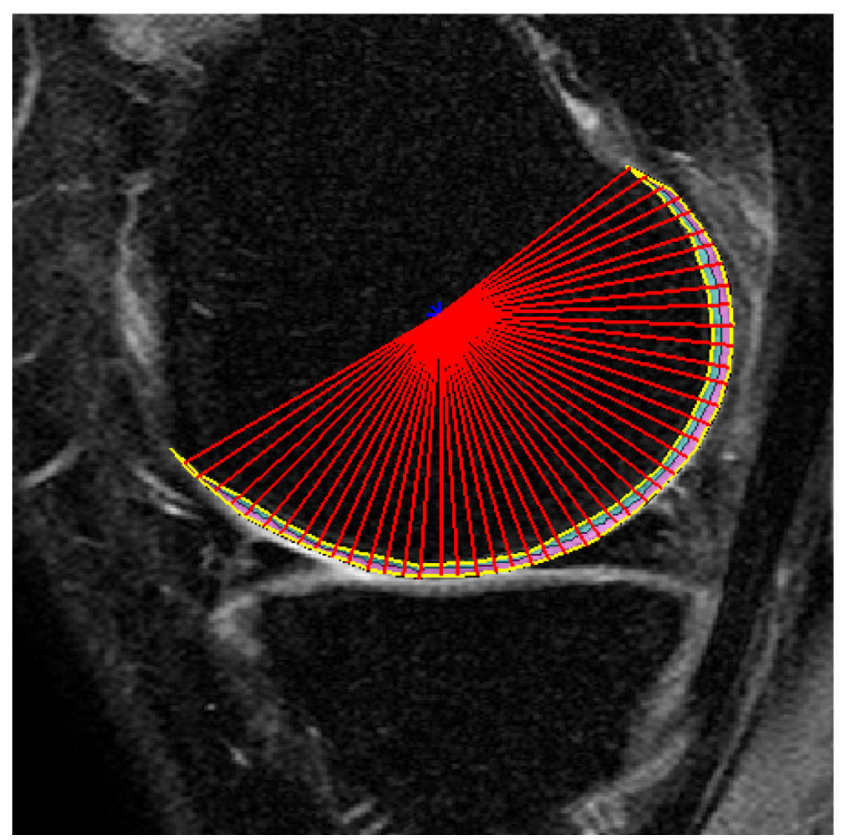

b-FFE

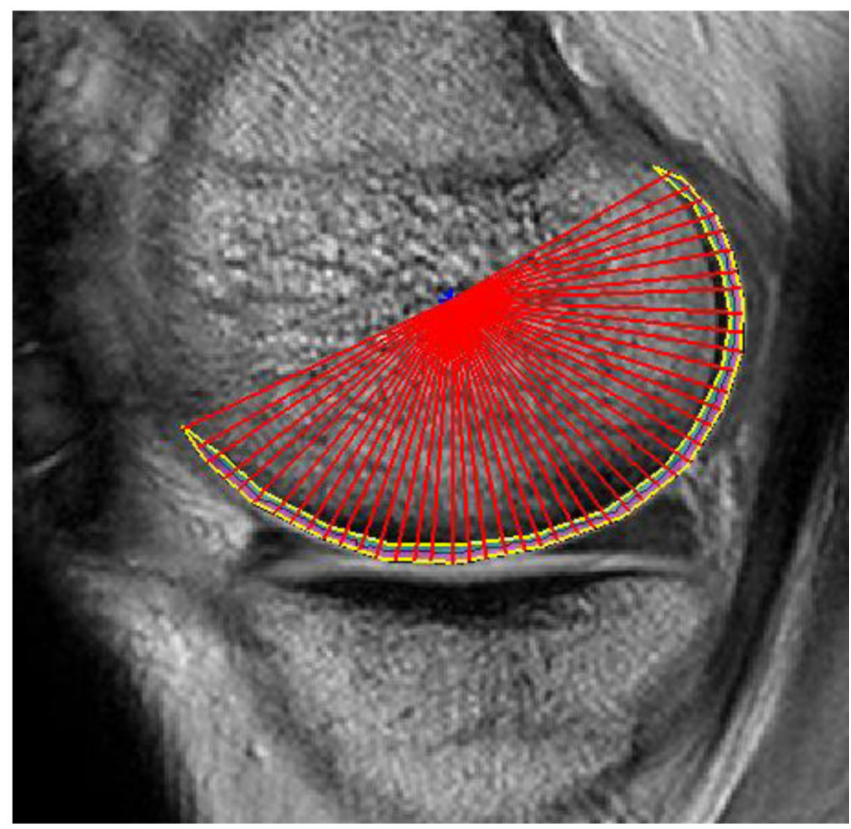

Figure1.

Sagittal images from T1rho sequence of knee MRI after manual segmentation with postprocessing.

(A) Spoiled gradient echo (SPGR) image. (B) Balanced steady state free precession (b-FFE) image. Two observers independently segmented the entire femoral cartilage of both images slice by slice. After manual segmentation, angular analysis in step of 4-degree and depth analysis with superficial and deep layers was performed automatically. 


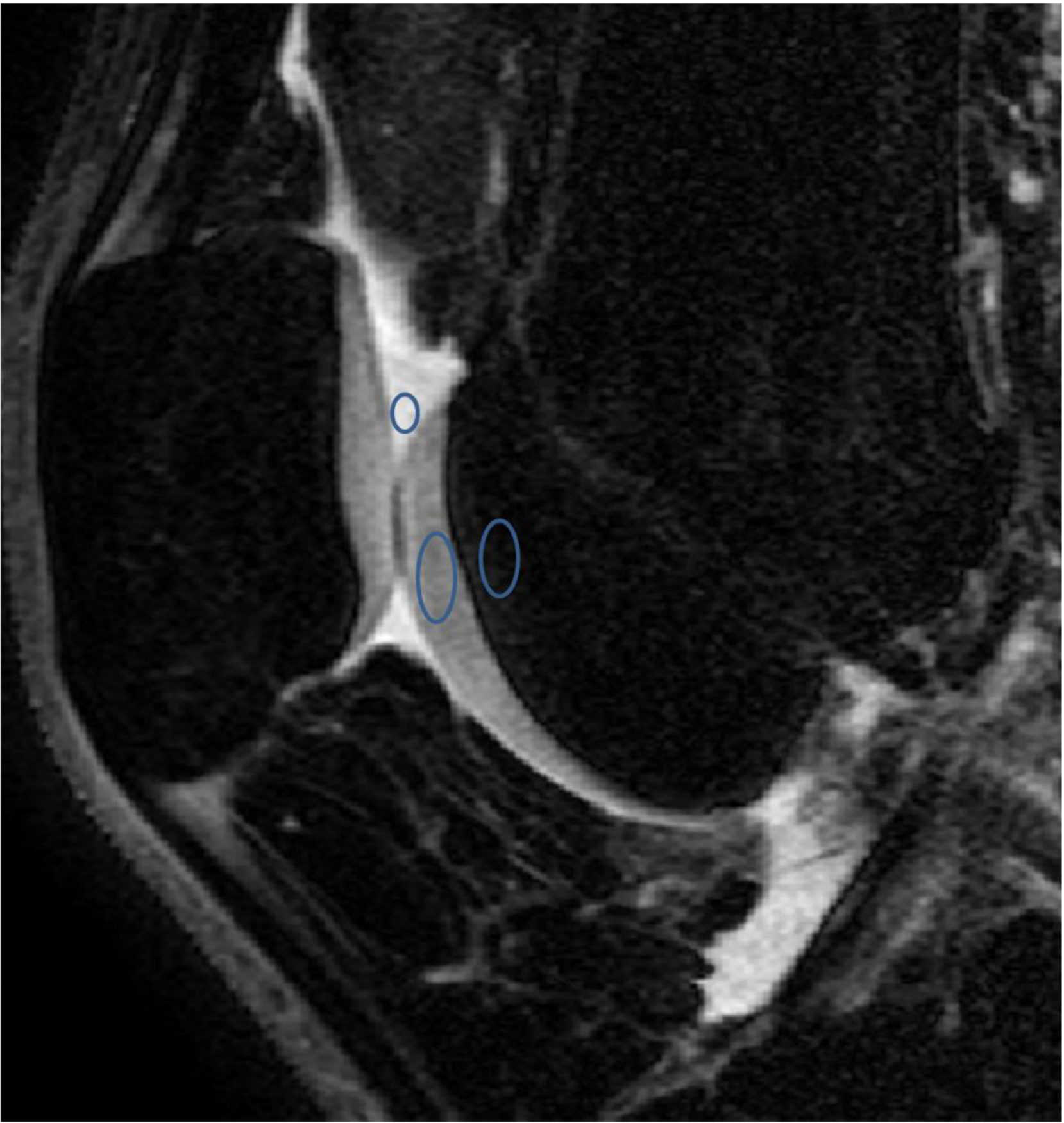

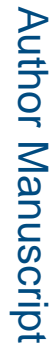

Figure2.

SPGR image demonstrating an example of ROIs on femoral cartilage (yellow circle), fluid in the suprapatellar bursa (red circle), and subchondral bone marrow in the femur (blue circle) for measurement of signal intensities. 


\section{Inter-, intraobserver reproducibility: SPGR}

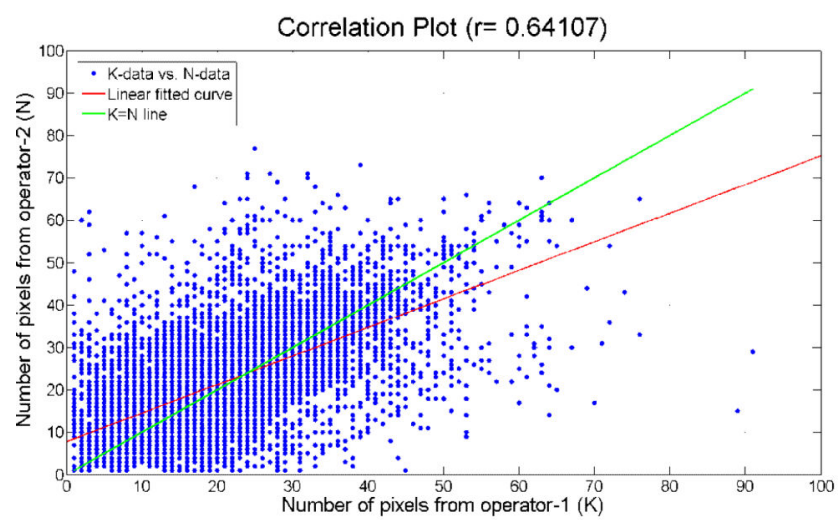

Whole layer (pixel numbers)-inter

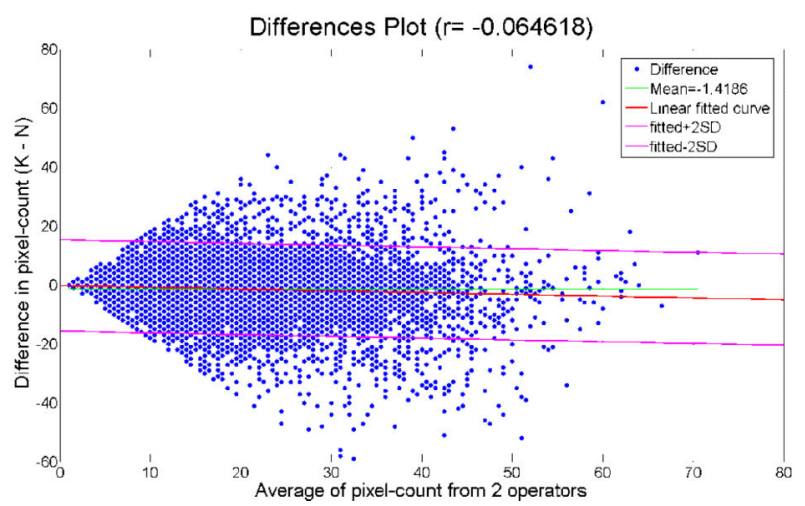

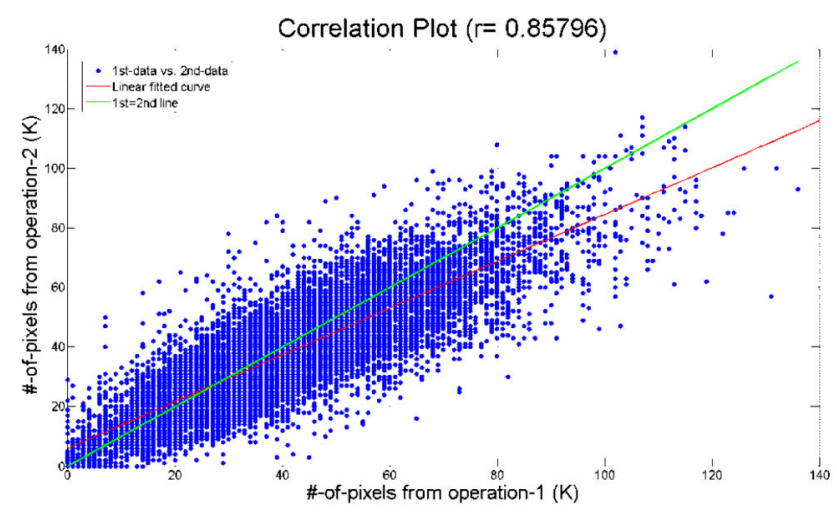

Whole layer (pixel numbers)-intra

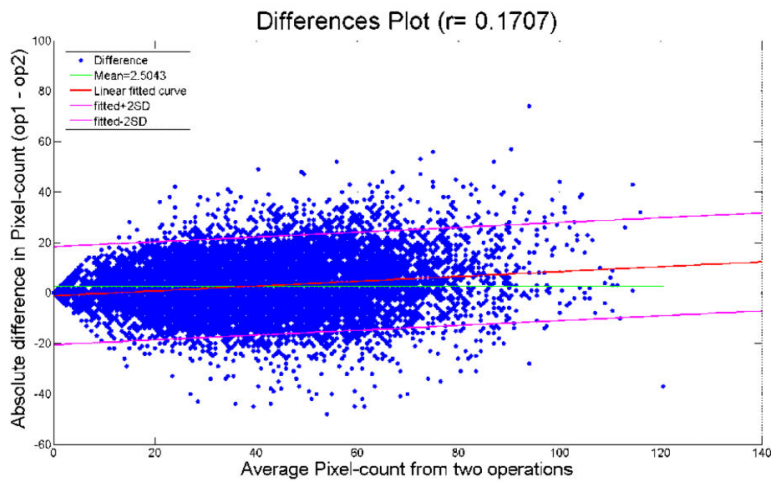

Figure3.

Bland-Altman plots of pixel counts in whole layer segmentation of the femoral cartilage on the SPGR sequence between inter- and intra-observer. (A) Correlation plot of inter-observer agreement (B) Difference plot of inter-observer agreement (C) Correlation plot of intraobserver agreement (D) Difference plot of intra-observer agreement. 


\section{Inter-, intraobserver reproducibility: b-FFE}

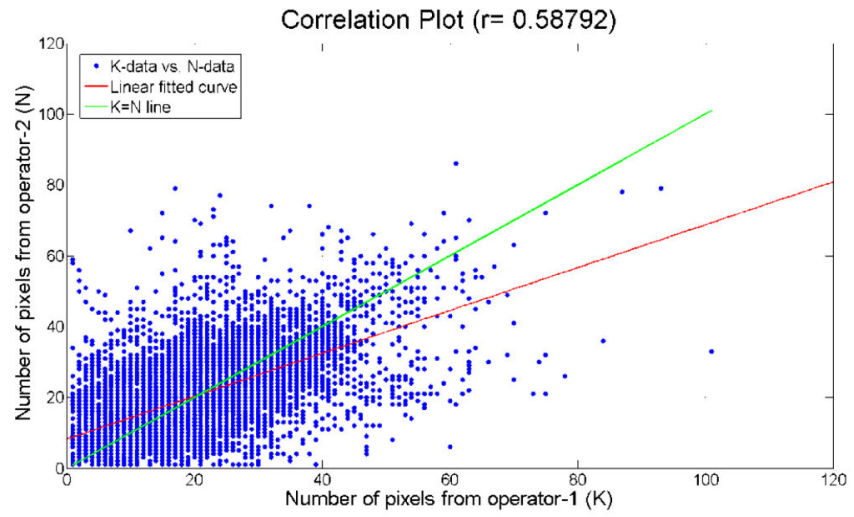

Whole layer (pixel numbers)-inter

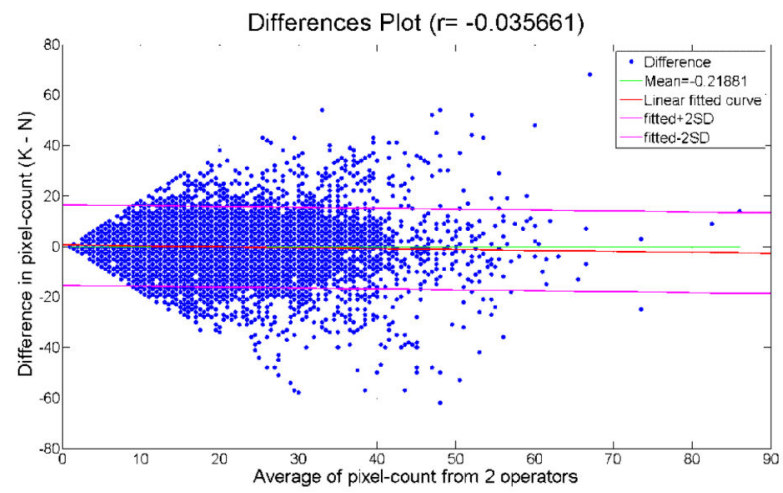

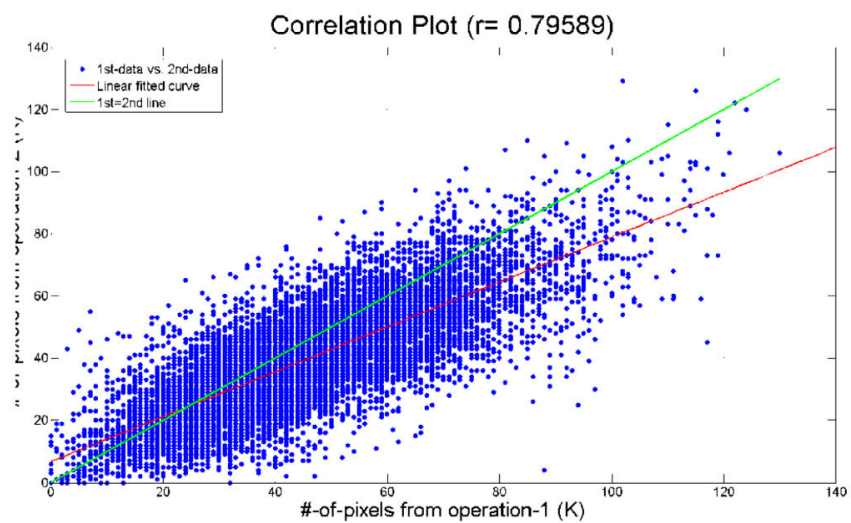

Whole layer (pixel numbers)-intra

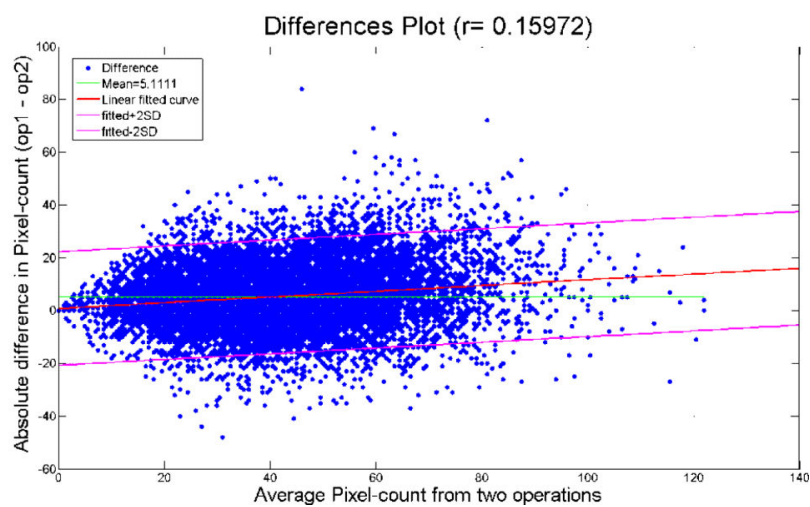

Figure4.

Bland-Altman plots of pixel counts in whole layer segmentation of the femoral cartilage on b-FFE sequence between inter- and intra-observer. (A) Correlation plot of inter-observer agreement (B) Difference plot of inter-observer agreement (C) Correlation plot of intraobserver agreement (D) Difference plot of intra-observer agreement. 
A

Relative SI

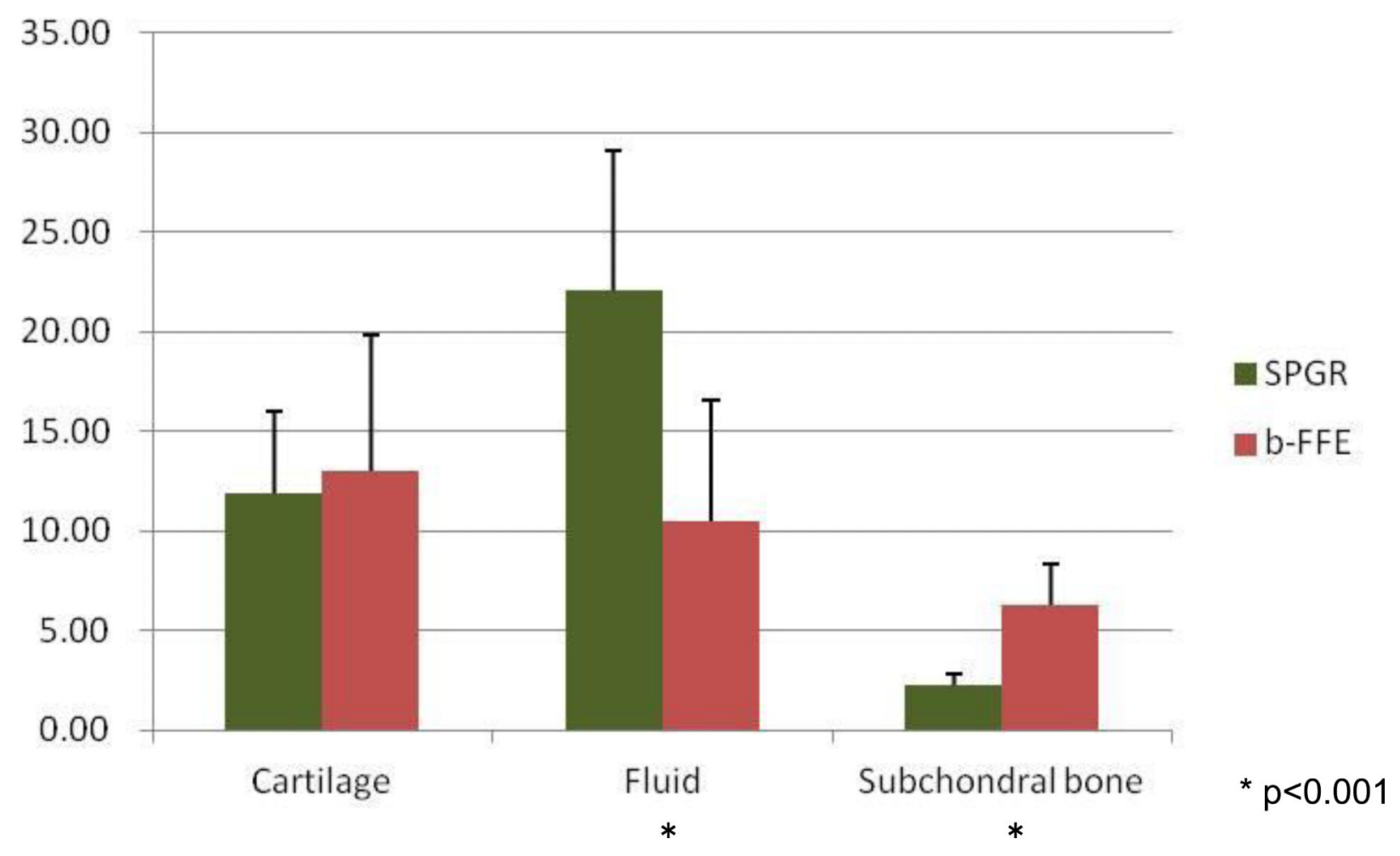


B

\section{Relative Contrast}

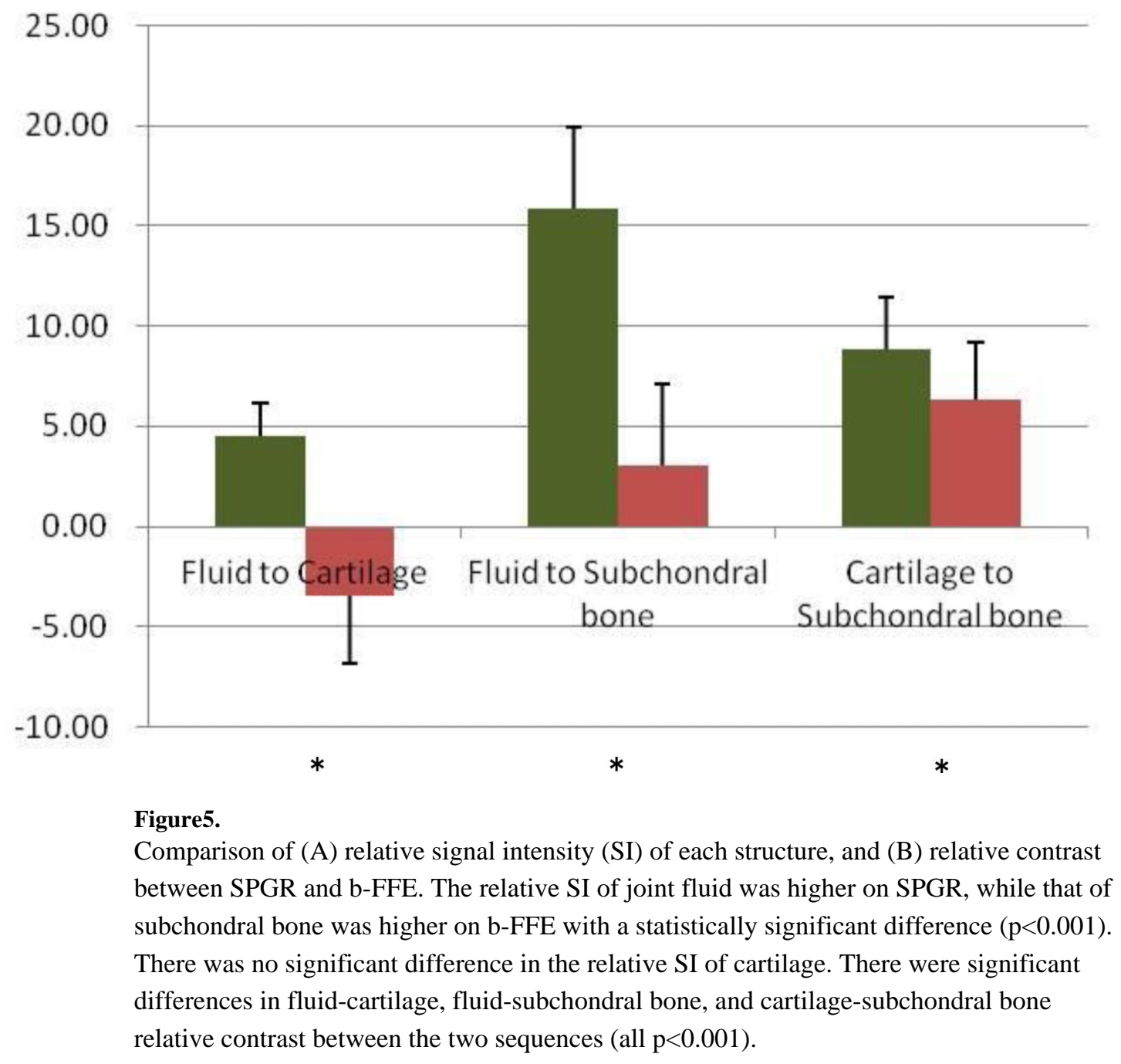

${ }^{*} p<0.001$

SPGR

b-FFE 
A

Comparison of T1rho value of entire femoral cartilage between SPGR and b-FFE: superficial layer

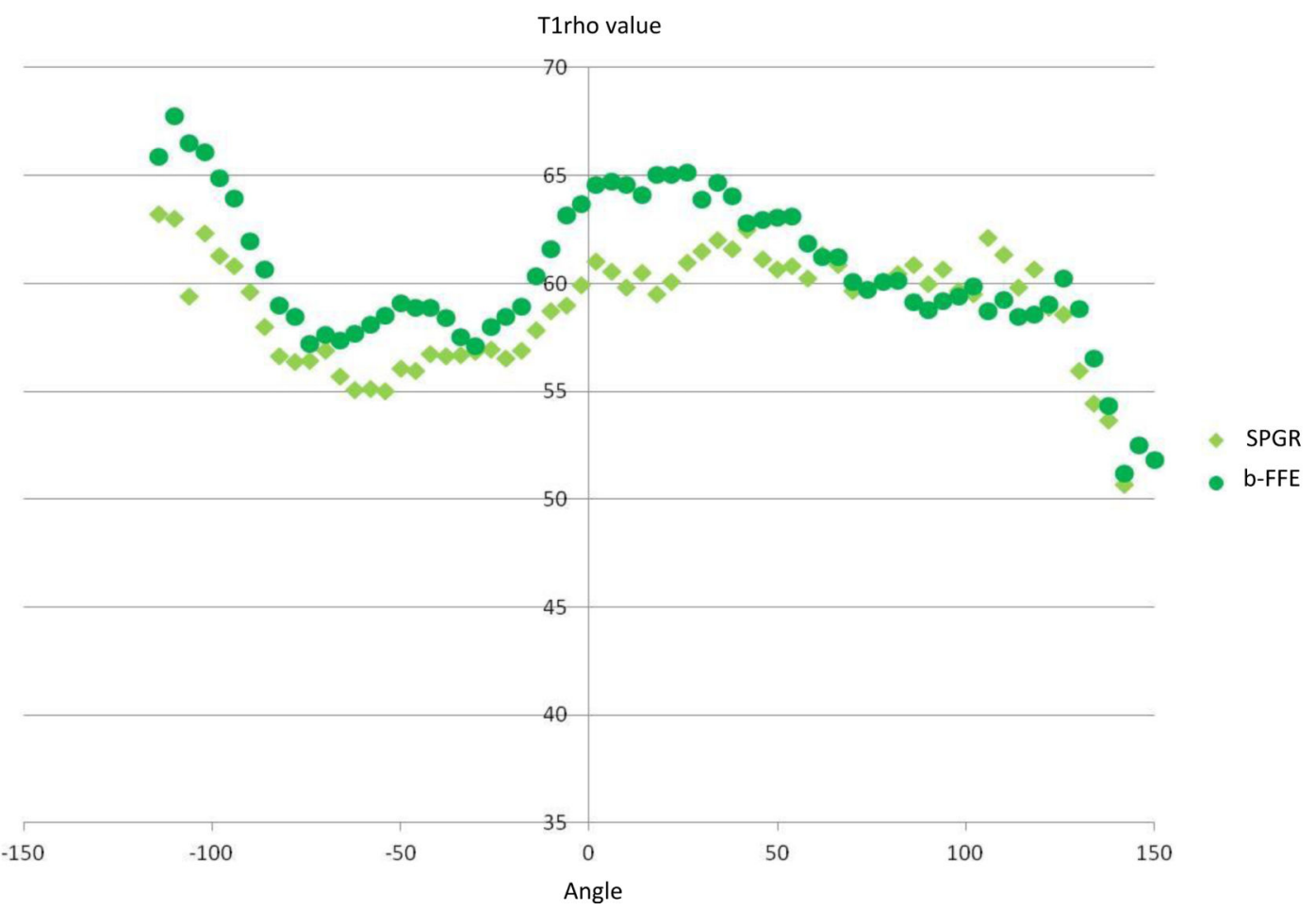


B

Comparison of T1rho value of entire femoral cartilage between SPGR and b-FFE: deep layer

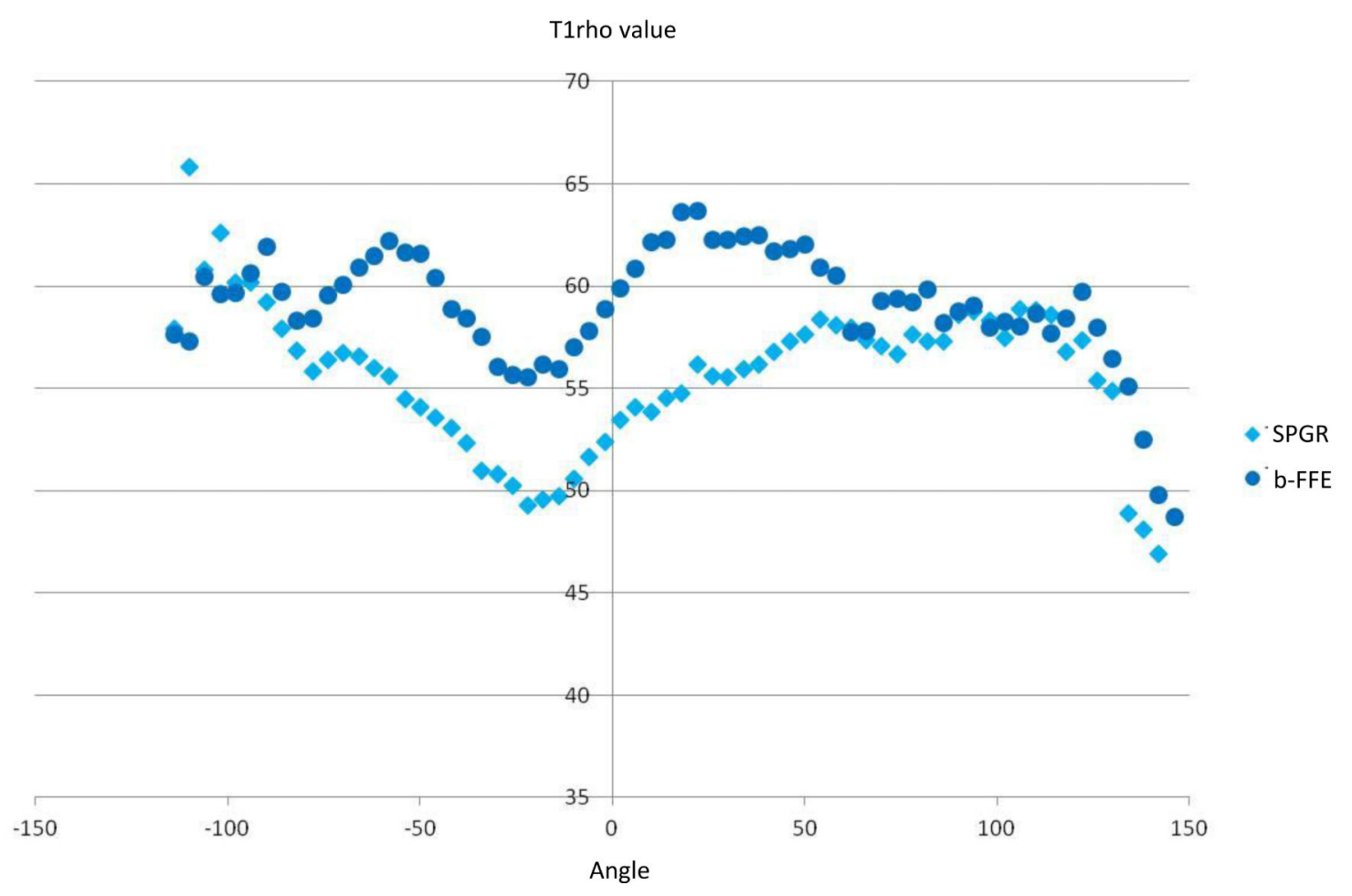




\section{Comparison of T1rho value of entire femoral cartilage between SPGR and b-FFE: Whole layer}

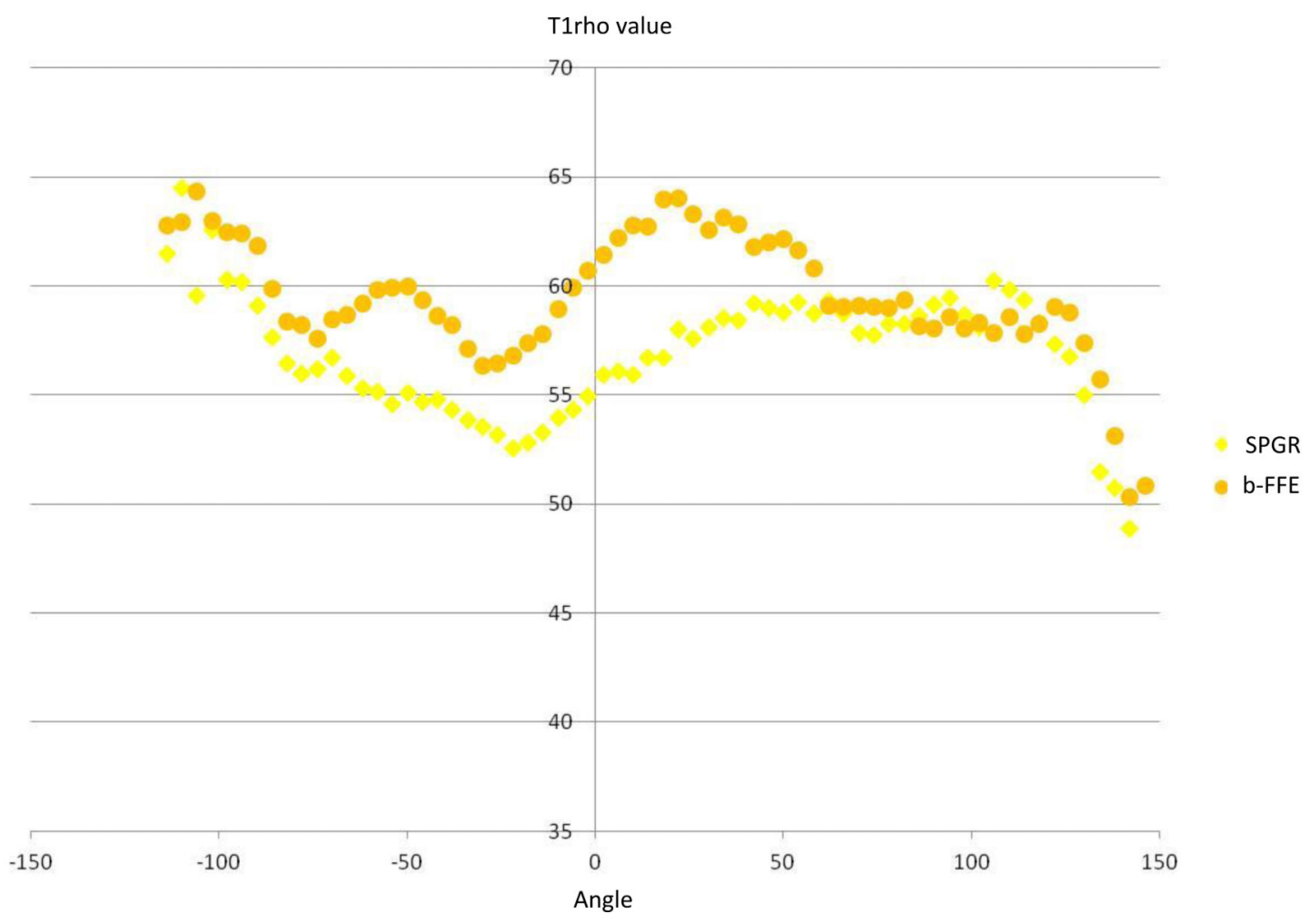

Figure6.

Comparison of T1rho values of the entire femoral cartilage between SPGR and b-FFE. (A) superficial layer. (B) deep layer. (C) whole layer. T1rho values tend to be higher on the bFFE sequence than on the SPGR sequence in all layers. There exists angular dependence of T1rho profiles in each layer on both sequences. 

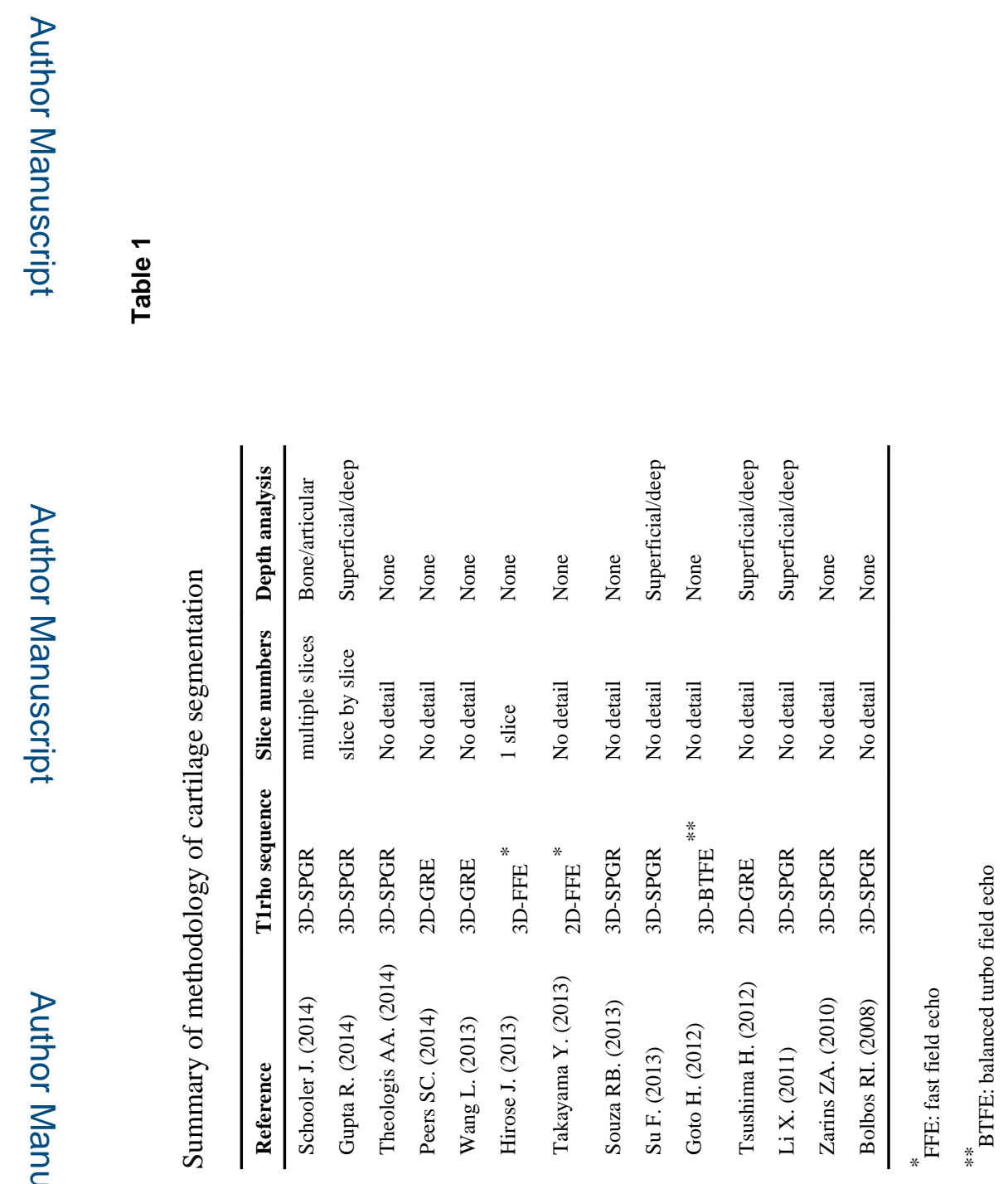


\section{롤 \\ 일}

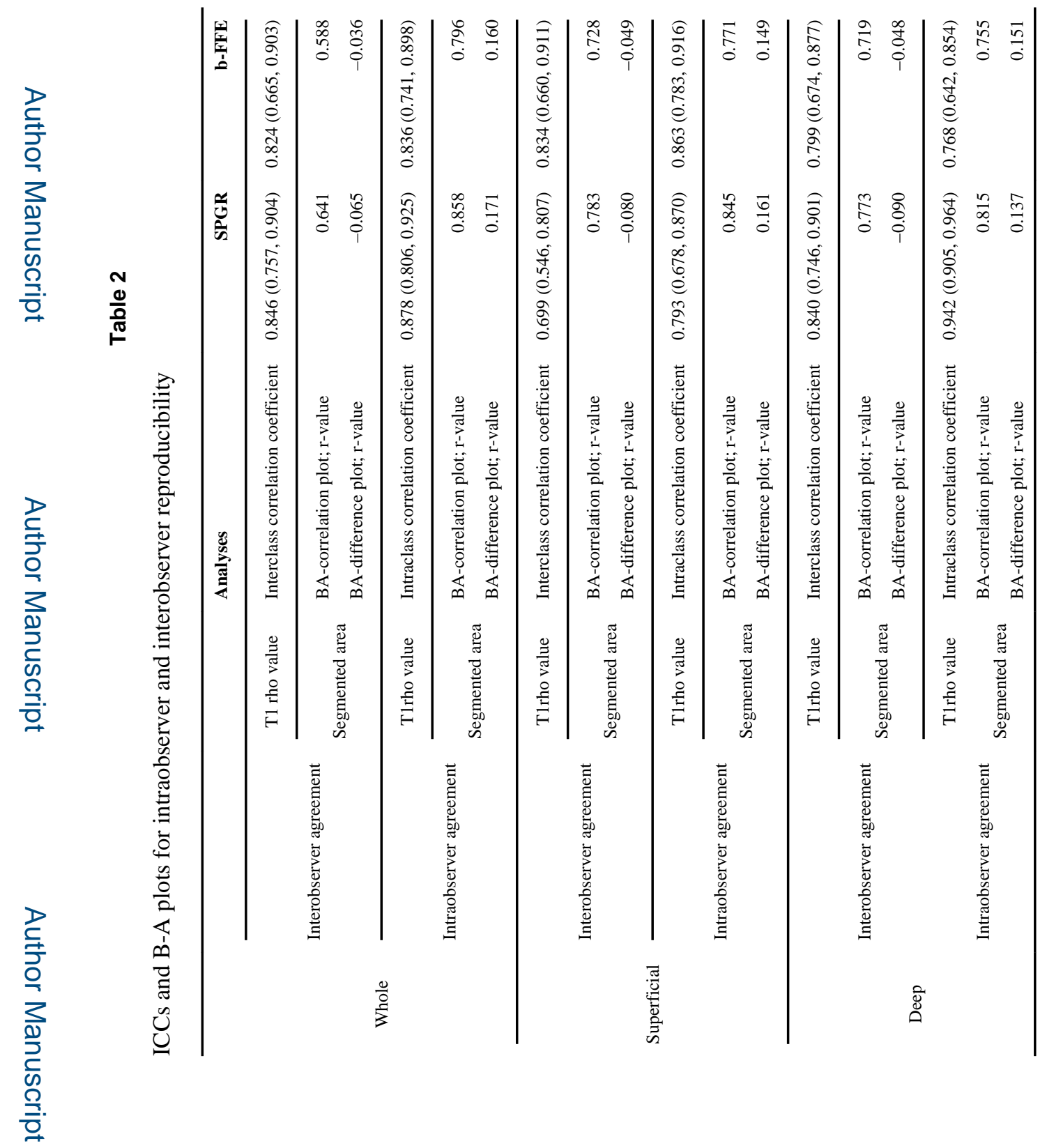

Eur J Radiol. Author manuscript; available in PMC 2016 January 14. 